\title{
Schisandra chinensis and $R$ hodiola rosea exert an anti-stress effect on the HPA axis and reduce hypothalamic c-Fos expression in rats subjected to repeated stress
}

\author{
NAN XIA ${ }^{1}$, JIE LI $^{1}$, HONGWEI WANG $^{2}$, JIAN WANG $^{1}$ and YANGTIAN WANG ${ }^{1}$ \\ ${ }^{1}$ Department of Endocrinology, Jinling Hospital; ${ }^{2}$ Jiangsu Key Laboratory for Molecular Medicine, School of Medicine, \\ Nanjing University, Nanjing, Jiangsu 210002, P.R. China
}

Received October 13, 2014; Accepted October 23, 2015

DOI: $10.3892 /$ etm.2015.2882

\begin{abstract}
The aim of the present study was to investigate the effects of Schisandra chinensis (S. chinensis) and Rhodiola rosea (R. rosea) on rats subjected to $5 \mathrm{~h}$ of stress, induced by water-floating followed by treadmill exercise. Hypothalamus-pituitary-adrenal (HPA) activity and c-Fos and Fos-related antigen 2 (Fra-2) mRNA expression levels in the hypothalamus of the rats were evaluated. Rats were distributed into four groups: $S$. chinensis $(n=12), R$. rosea $(n=10)$, stress control $(\mathrm{n}=10)$ and quiet control $(\mathrm{n}=8)$. Following a training period of 6 consecutive days, the $S$. chinensis, $R$. rosea and stress control groups underwent a 3 -h water-floating session in the presence of feline predators immediately followed by $2 \mathrm{~h}$ treadmill running to induce psychological and physical stress. Following compound stress induction, the serum levels of corticosterone (CORT), adrenocorticotropic hormone and interleukin-1 $\beta$ and the mRNA expression levels of hypothalamic corticotropin-releasing hormone (CRH), neuropeptide-Y, c-Fos and Fra-2 were evaluated using enzyme-linked immunosorbent assay, radioimmunoassay and quantitative polymerase chain reaction, respectively. The results indicated that $S$. chinensis and $R$. rosea markedly decreased the stress-induced elevation of CRH and peripheral CORT levels. The mRNA expression levels of c-Fos and Fra-2 in the hypothalamus were significantly increased after $5 \mathrm{~h}$ compound stress, and reduced levels of c-Fos expression were detected in rats treated with $R$. rosea. Thus, $S$. chinensis and $R$. rosea exert an anti-stress effect in rats subjected to stress by balancing the HPA axis, and possibly by reducing the expression of c-Fos in the hypothalamus.
\end{abstract}

Correspondence to: Dr Jian Wang or Dr Yangtian Wang, Department of Endocrinology, Jinling Hospital, 305 East Zhongshan Road, Nanjing, Jiangsu 210002, P.R. China

E-mail: wangjndcrn@aliyun.com

E-mail: sky16@vip.sina.com

Key words: Schisandra chinensis, Rhodiola rosea, hypothalamus-pituitary-adrenal axis, immediate early genes, stress

\section{Introduction}

Stress is caused by a variety of stressors, that is, harmful stimuli or challenges that lead to various physiological, behavioral, emotional and cognitive alterations known as stress responses. It is an adaptive response mediated by the stress system, which includes the central nervous system and peripheral components in various physiological and pathological states. The primary components of the stress system are corticotropin-releasing hormone (CRH), also known as corticotropin-releasing factor, locus coeruleus-norepinephrine-autonomic systems and their peripheral effectors, the hypothalamus-pituitary-adrenal (HPA) axis and the limbs of the autonomic system (1-3). Stress responses are highly organized and regulated, and act to reduce the adverse impact of a stressor. By contrast with the view that the stress response restores the stability of an organism's internal environment (homeostasis), it is now hypothesized that stressors cause well-organized responses with their own homeostasis, to promote adaptive coping (1). These adaptive changes are usually time-limited, which may improve an individual's survivability. However, when stress is severe or chronic, or there is a functional defect of inadequate response to stress, the organization and regulation of stress responses may be disrupted, resulting in various endocrine, metabolic, autoimmune and psychiatric disorders $(1,2)$. Therefore, to maintain a healthy state, it is crucial to limit the extent of harmful external stimuli, reduce the adverse effects of severe or chronic stress and enhance the body's resistance to stressors.

Following acute exposure to stressors, including fatigue, heat shock, skin burn, cooling, frostbite, immobilization and swimming under load, the amplitude and synchronization of the CRH and arginine-vasopressin pulsations in the hypophyseal portal system markedly increase, causing adrenocorticotropic hormone (ACTH) and cortisol secretion levels to increase (4). CRH serves a crucial function in the activation of the stress response. A circumscribed group of parvocellular neurosecretory neurons in the paraventricular nuclei (PVN) of the hypothalamus is the principal source of $\mathrm{CRH}$ for delivery to the portal capillary zone of the median eminence, although $\mathrm{CRH}$ is broadly expressed throughout the central nervous system $(5,6)$. Glucocorticoids, as the final effectors of the HPA axis, help to maintain homeostasis and the body's response 
to stress, resulting in behavioral and peripheral changes that improve the ability to adjust to external challenges.

Adaptogens augment resistance to stress and increase concentration, performance and fatigue endurance (7-10). In the 1960 s, on the basis of the results of numerous pharmacological and clinical studies, three plant species, namely Eleutherococcus senticosus, Rhodiola rosea (R. rosea) and Schisandra chinensis (S. chinensis), were incorporated into official medical practice in the Union of Soviet Socialist Republics as adaptogens, and were considered to exert stimulatory, restorative and anti-stress effects (10). These plants may be used by healthy individuals as tonics during states of fatigue and stress, and in sports medicine for preventing and treating injuries. In addition, they have been employed in occupational medicine for protection against adverse environmental factors, including extreme conditions such as high noise levels and low temperature, and in clinical medical practice for treating acute hepatic poisoning, ischemia due to oxygen deprivation and for accelerating recovery following surgery.

As medicinal plants, $R$. rosea and $S$. chinensis appear to possess the characteristics of adaptogens (10-13), which were described by Brekhman (14) as follows: i) Produces a non-specific response in an organism, for example, an increase in power of resistance against multiple stressors, including physical, chemical or biological agents; ii) has a normalizing influence on physiology, irrespective of the direction of change from physiological norms caused by the stressor; and iii) is incapable of influencing normal body functions more than required to gain non-specific resistance. By definition, adaptogens reinforce the non-specific resistance against stressors, increase the capacity to withstand stress, and thus protect against disease caused by excessive stress. Plant adaptogens do not exert unwanted effects on normal physiological functions (15). By contrast, stimulants such as caffeine and nicotine that increase alertness and concentration by enhancing the activity of the sympathetic nervous system, may possess addictive, tolerance and abuse potential and their long-term use may impair mental function and result in psychiatric disorders (15).

Previous studies have shown that ADAPT-232 forte, which comprises $E$. senticosus, $S$. chinensis and $R$. rosea extracts, prolongs the period until exhaustion by $\sim 7$-fold and repeated administration of this adaptogen may increase basal levels of heat shock protein 72 (Hsp72) in the serum of mice (16). A number of other stress-related factors have been extensively studied in the process of stress, including neuropeptide-Y (NPY) (17,18), Hsp70, activating protein-1 (AP-1) and cytokines such as interleukin (IL)-1 $\beta$, IL-6 and tumor necrosis factor- $\alpha$ (TNF- $\alpha$ ) (19). In addition, the rapid induction of immediate early genes such as c-Fos, Fos-related antigen (Fra)-1, Fra-2 and c-Jun may alter gene transcription in hormone-secreting cells in the hypothalamus, pituitary gland and adrenal gland, which may result in changes in behavior, morphology, and the survival or degeneration of neurons in response to pathophysiological stimuli (19). Previous studies have shown that c-Fos in the hypothalamus is a reliable marker for activated cells and central nervous system circuits that respond to stress challenges (20-25). Furthermore, Fra-2 mRNA expression has been detected in the hippocampus (26), and the suprachiasmatic nucleus and PVN in the hypothalamus during stress. These stress-related factors have been demonstrated to be crucially involved in the mediation of the stress process.

In a previous study, we established an acute stress model in which adult rats were subjected to water-floating and high-intensity exercise, with the aim of simulating psychological and physical stress, respectively (27). The aim of the present study was to investigate the effects of $S$. chinensis and $R$. rosea in rats subjected to acute compound stress, and to determine the underlying mechanisms by evaluating the serum levels of ACTH, CORT and IL-1 $\beta$ and the mRNA expression levels of $\mathrm{CRH}$, NPY, c-Fos and Fra-2 in the whole hypothalamus of the rats exposed to $S$. chinensis and $R$. rosea.

\section{Materials and methods}

Animals. A total of 40 male Sprague-Dawley rats (age, 10 weeks; weight, $300 \pm 20 \mathrm{~g}$ ) were purchased from the Shanghai SLAC Laboratory Animal Co. Ltd. (Shanghai, China). Prior to the study, the rats were housed for 3 days in a clean-grade animal breeding center with an indoor temperature of $20-24^{\circ} \mathrm{C}$ and humidity of $50-70 \%$, under alternate dark/light cycles. Tap water and laboratory feed were available ad libitum. The rats were then allocated at random into four groups: S. chinensis $(\mathrm{n}=12), R$. rosea $(\mathrm{n}=10)$, stress control $(\mathrm{n}=10)$ and quiet control $(\mathrm{n}=8)$. All procedures were performed in accordance with the standards of the Animal Research Ethics Committee of Jinling Hospital (Nanjing, China).

Water-floating and treadmill training. Four identical plastic containers $(1.00 \times 0.67 \times 0.60 \mathrm{~m})$ were used as floating devices and were placed in an inflatable swimming pool $(3.8 \times 5 \mathrm{~m})$ in which a wave of $15-20^{\circ}$ was produced using three water pumps (Shenzhen XingRisheng Industrial Co., Ltd., Shenzhen, China). Three-year-old tabby cats, weighing $\sim 5 \mathrm{~kg}$, were purchased from a local pet store, fed feline food, and maintained in a cage in a clean-grade room with an alternate light/dark cycle at $20-24^{\circ} \mathrm{C}$ and $50-70 \%$ humidity. According to the feline predator stress model proposed by Adamec et al (28), on days 1-6 at 13:00, all rats (including the quiet control group) were placed on the floating container for $10 \mathrm{~min}$, and simultaneously stimulated by the threat of two cats located in cages at a distance of $0.75 \mathrm{~m}$. The floating was immediately followed by 10 min treadmill training, which provided high-intensity exercise using an ZH-PT rat treadmill (Huaibei Zhenghua Bioinstrumentation Co., Ltd., Huaibei, China) with a $0^{\circ}$ slope at a speed of $15 \mathrm{~m} / \mathrm{min}$. All rats successfully completed the training and so none were eliminated from the study. During this 6-day training period, S. chinensis and $R$. rosea were administered by gavage twice a day to rats in the corresponding intervention groups. The dosages of each were $1 \mathrm{mg} / \mathrm{kg}$ each time, according to preliminary experiments. The rats in the stress control group and quiet control group were administered identical quantities of normal saline. S. chinensis and $R$. rosea used in the study were provided by Department of Traditional Chinese Medicine of Jinling Hospital.

Exhaustion experiment (compound stress model). At 7:00 a.m. on day 7 , the rats in the $S$. chinensis, $R$. rosea and stress control groups underwent water-floating for $3 \mathrm{~h}$ with cats at a distance of $0.75 \mathrm{~m}$, while the quiet control rats remained in their cages. Next, the three water-floating groups underwent high-intensity 
exercise on a treadmill with a $5^{\circ}$ slope at $26.8 \mathrm{~m} / \mathrm{min}(27)$. According to the study protocol, rats that showed signs of exhaustion during the exercise or that were unable to maintain the required speed were immediately anaesthetized with $50 \mathrm{mg} / \mathrm{kg}$ sodium pentobarbital and sacrificed via decapitation. In the present study, all rats successfully completed the 2-h treadmill exercise, with 3 exceptions; 1 rat in the $S$. chinensis group, 1 rat in the $R$. rosea group and 1 rat in the stress control group. All rats were deprived of food and water following the initiation of the water-floating experiment.

Blood sampling. Following the treadmill exercise, all rats were anaesthetized with $50 \mathrm{mg} / \mathrm{kg}$ sodium pentobarbital dissolved in normal saline and sacrificed via decapitation. Within $30 \mathrm{~min}$, $5 \mathrm{ml}$ blood was harvested, the hair and skull at the back of the head were carefully removed and the hypothalamus was isolated using tweezers. Hypothalamic tissue was immediately immersed in liquid nitrogen and stored at $-80^{\circ} \mathrm{C}$ for subsequent reverse transcription-quantitative polymerase chain reaction (RT-qPCR) assays to evaluate the mRNA expression levels of CRH, NPY, c-Fos and Fra-2. Serum was isolated for the subsequent measurement of ACTH and IL-1 $\beta$ concentrations.

CORT enzyme-linked immunosorbent assay (ELISA). CORT concentration was measured using a CORT ELISA kit (assay sensitivity of 46.88 pg/ml; Elabscience Biotechnology Co., Ltd., Wuhan, China) according to the manufacturer's instructions. Briefly, serum samples were added to 96 -well plates containing biotinylated primary antibody and incubated at $37^{\circ} \mathrm{C}$ for $45 \mathrm{~min}$. Plates were washed and horseradish peroxidase-conjugated streptavidin solution was added to the wells and incubated for a further $30 \mathrm{~min}$ at $37^{\circ} \mathrm{C}$. The plates were then washed, 3,3',5,5'-tetramethylbenzidine substrate was added and the plates were incubated for an additional $15 \mathrm{~min}$ at $37^{\circ} \mathrm{C}$. Finally, stop solution was added to the wells to terminate the reaction. The resultant absorbance was measured at $450 \mathrm{~nm}$ using an iMark microplate reader (Bio-Rad Laboratories, Inc., Hercules, CA, USA). The concentration of CORT was determined using a standard curve.

Radioimmunoassay. Serum ACTH and IL-1 $\beta$ concentrations were measured using radioimmunoassay testing kits (North Institute of Biological Technology, Beijing, China), according to the manufacturer's instructions. Assay sensitivities for ACTH and IL-1 $\beta$ were $<0.1 \mathrm{ng} / \mathrm{ml}$ and $<5 \mathrm{pg} / \mathrm{ml}$, respectively, for a 100- $\mu 1$ sample. The detection limit for ACTH was $\sim 405 \mathrm{pg} / \mathrm{ml}$.

$R N A$ isolation and RT-qPCR. Frozen hypothalamic tissue was punched and ground into pellets using a mortar and pestle so that the pellets were as small as possible. RNA was isolated and subsequently purified from the punched pellets of hypothalamic tissue using an RNAprep Micro Sample RNA Extraction kit, according to the manufacturer's protocol (BioTeke Corporation, Beijing, China). Following the isolation and purification of the total RNA, the concentration of each individual total RNA sample was standardized as $250 \mathrm{ng} / \mathrm{ml}$. To generate single-strand cDNA, total RNA was used as the starting template for first strand cDNA synthesis, using a PCR RevertAid First strand cDNA Synthesis kit (Thermo Fisher Scientific, Waltham, MA, US) according to the manufacturer's instructions. Subsequently,
Table I. Primer sequences for polymerase chain reaction.

\begin{tabular}{lll}
\hline Gene & Direction & \multicolumn{1}{c}{ Primer $\left(5^{\prime}-3^{\prime}\right)$} \\
\hline CRH & Forward & ATCTCACCTTCCACCTTCTG \\
& Reverse & GCAACATTTCATTTCCCGATAATC \\
NPY & Forward & ACAGAGATATGGCAAGAGA \\
& Reverse & CACAGGATGAGATGAGATG \\
c-Fos & Forward & GGGAGGACCTTATCTGTGCG \\
& Reverse & TCTCCGGAAGAGGTGAGGAC \\
Fra-2 & Forward & AACCTTGTCTTCACCTACC \\
& Reverse & CCACTGCTACTGCTTCTG
\end{tabular}

CRH, corticotropin-releasing hormone; NPY, neuropeptide-Y; Fra-2, Fos-related antigen 2.

qPCR was performed using CFX connect Real-Time qPCR system (Bio-Rad Laboratories, Inc.) and a SuperReal PreMix Plus (SYBR Green; Qiagen, Inc., Valencia, CA, USA) kit. The PCR primers used for each gene are presented in Table I. The qPCR reaction was performed using a final reaction volume of $20 \mu \mathrm{l}$, which included $10 \mu \mathrm{mol} / \mu \mathrm{l}$ of each primer, $10 \mu 1$ SuperReal PreMix Plus (SYBR Green) and $8 \mu 1$ template. Samples were subjected to a 3-min incubation at $95^{\circ} \mathrm{C}$, followed by 40 cycles of $10 \mathrm{sec}$ at $95^{\circ} \mathrm{C}, 20 \mathrm{sec}$ at $59^{\circ} \mathrm{C}$ and $30 \mathrm{sec}$ at $72^{\circ} \mathrm{C}$. The PCR products were subjected to melt curve analysis to exclude the possibility of the amplification of unwanted products. Results were analyzed using CFX Manager software (Bio-Rad Laboratories, Inc.).

Statistical analysis. All measurement data are expressed as the mean \pm standard error of the mean. Absolute measurement data of hormone concentrations are provided; however, gene expression data are expressed as arbitrary optical density units. One-way analysis of variance was used to determine the significance of the effects of the adaptogen treatment on hormone and gene expression. If a significant interaction was detected, post hoc Dunnet's t-tests were performed. All stressed groups were compared with the control group using the one-tailed t-test on the basis of the a priori hypothesis that stress increases the levels of hormones and mRNA expression. $\mathrm{P}<0.05$ was considered to indicate a statistically significant difference.

\section{Results}

HPA axis activity. The mRNA expression levels of CRH in the hypothalamus were significantly higher in the stress control group compared with the quiet control group $(\mathrm{P}<0.05)$. No significant differences in the serum levels of CORT and ACTH were detected between the stress control and quiet control groups following the water-floating and exhaustion experiments. The expression levels of CRH mRNA in the hypothalamus and of CORT in the serum were significantly reduced in the $S$. chinensis and $R$. rosea groups compared with those in the stress control group following the exhaustion experiment $(\mathrm{P}<0.05$; Fig. 1). 

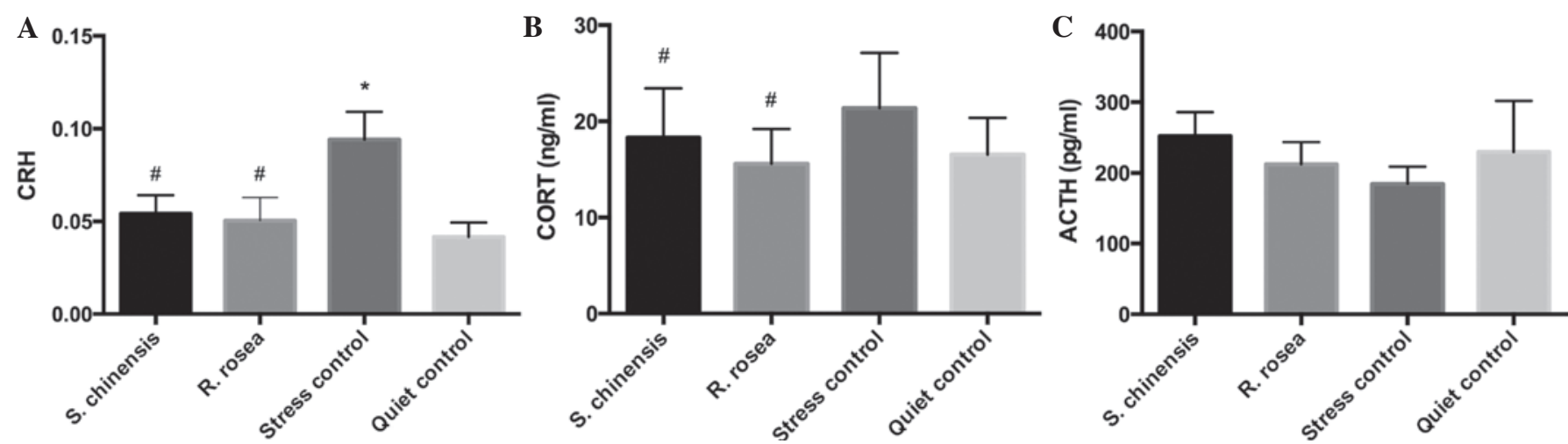

Figure 1. Comparison of the hypothalamus levels of CRH and the serum levels of CORT and ACTH between the four groups. (A) The mRNA expression levels of $\mathrm{CRH}$ in the hypothalamus were significantly increased in the stress control group compared with the quiet control following the water-floating and exhaustion experiments. By contrast, the serum levels of (B) CORT and (C) ACTH showed no significant difference. Compared with stress control group, the mRNA expression levels of $\mathrm{CRH}$ in the hypothalamus and the serum level of CORT were significantly reduced in $S$. chinensis and $R$. rosea groups. ${ }^{*} \mathrm{P} \leq 0.05$ vs. the quiet control; ${ }^{\text {P }} \leq 0.05$ vs. the stress control. $\mathrm{CRH}$, corticotropin-releasing hormone; $S$. chinensis, Schizandra chinensis; $R$. rosea, Rhodiola rosea; CORT, corticosterone; ACTH, adrenocorticotropic hormone.
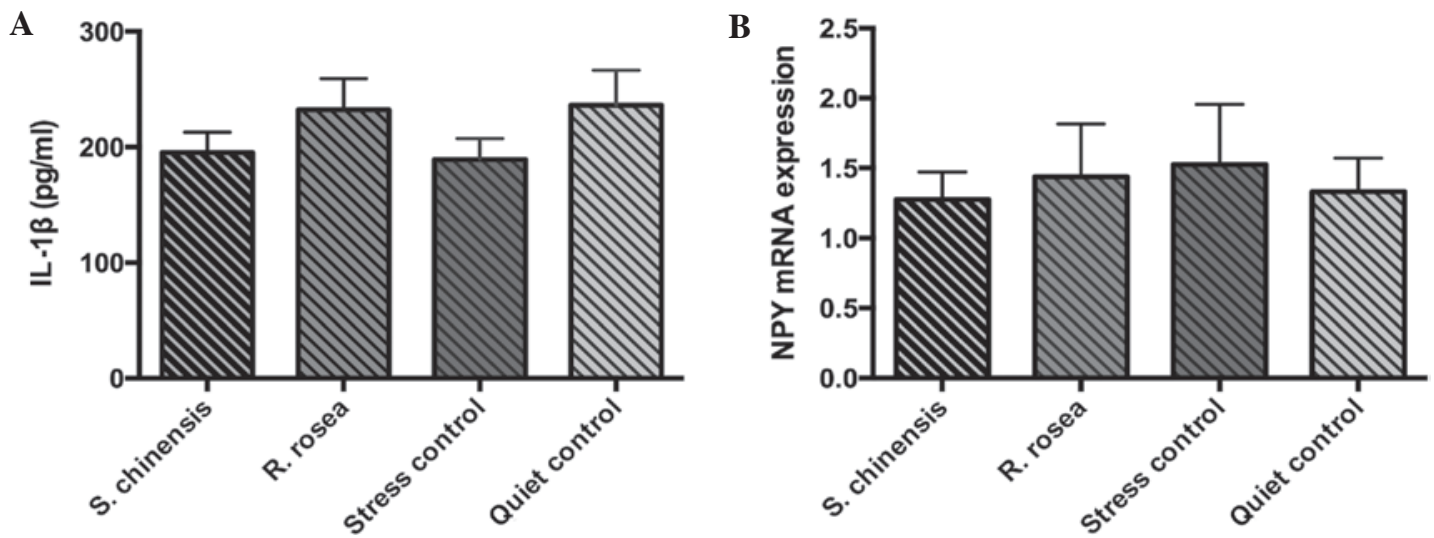

Figure 2. Comparison of serum IL- $\beta$ levels and hypothalamic NPY mRNA expression levels between the four groups. No significant differences were detected in the (A) serum IL-1 $\beta$ levels and (B) hypothalamic NPY mRNA expression levels between the rats in the four groups (P>0.05). IL-1 $\beta$, interleukin-1 $\beta$; S. chinensis, Schizandra chinensis; R. rosea, Rhodiola rosea; NPY, neuropeptide-Y.

Serum IL-1 levels and hypothalamic NPY mRNA expression. No significant differences in the serum IL-1 $\beta$ levels and hypothalamic NPY mRNA expression were identified between the four groups (P>0.05; Fig. 2).

c-Fos and Fra-2 mRNA expression in the hypothalamus. The c-Fos and Fra-2 mRNA expression levels in the hypothalamus of the stress control group were significantly higher compared with those of the quiet control $(\mathrm{P}<0.05)$. The c-Fos mRNA expression levels in the $S$. chinensis and $R$. rosea groups did not differ significantly compared with those in the quiet control group $(\mathrm{P}>0.05)$. The Fra-2 expression level in the S. chinensis and $R$. rosea groups did not significantly differ from the stress control group, but did show a significant difference compared with the quiet control group $(\mathrm{P}<0.05$; Fig. 3$)$.

\section{Discussion}

In the present study, a 3-h water-floating experiment including a feline predator stress element, and a 2-h treadmill exercise were conducted as psychological and physical stressors, respectively. Prior to the initiation of the experiment, all rats underwent 6 days of training to enable them to adapt to the long-term and intense stimulations on day 7. Following the experiment, serum levels of CORT and ACTH were determined, in addition to the $\mathrm{CRH}$ mRNA expression levels in the hypothalamus. CRH mRNA expression was significantly increased following exposure to prolonged compound stress; however, no significant differences in ACTH and CORT levels were observed. Furthermore, ACTH levels appeared to decrease slightly following the compound stress. It has been reported that the magnitude and duration of increases in serum ACTH concentration may be associated with the duration and intensity of the ACTH-releasing stimulus, and that elevated levels of ACTH persist throughout a 2-h period of ether stress ( $>700 \mathrm{pg} / \mathrm{ml}$ ) (29). By contrast, ACTH levels have been shown to reduce markedly under prolonged mobilization stress, with a reduction to almost twice the basal levels after $6 \mathrm{~h}$, from $>600 \mathrm{pg} / \mathrm{ml}$ to $<50 \mathrm{pg} / \mathrm{ml}$; these levels persisted for the remainder of the stress period (30). Previous results suggest that the elevation in plasma CORT levels in response to stress is biphasic, whereas plasma ACTH levels exhibit only a single peak that rapidly declines to a sustained plateau level (31). Plasma levels of CORT, but not those of ACTH, have been found to remain elevated after $180 \mathrm{~min}$ restraint (32). In 

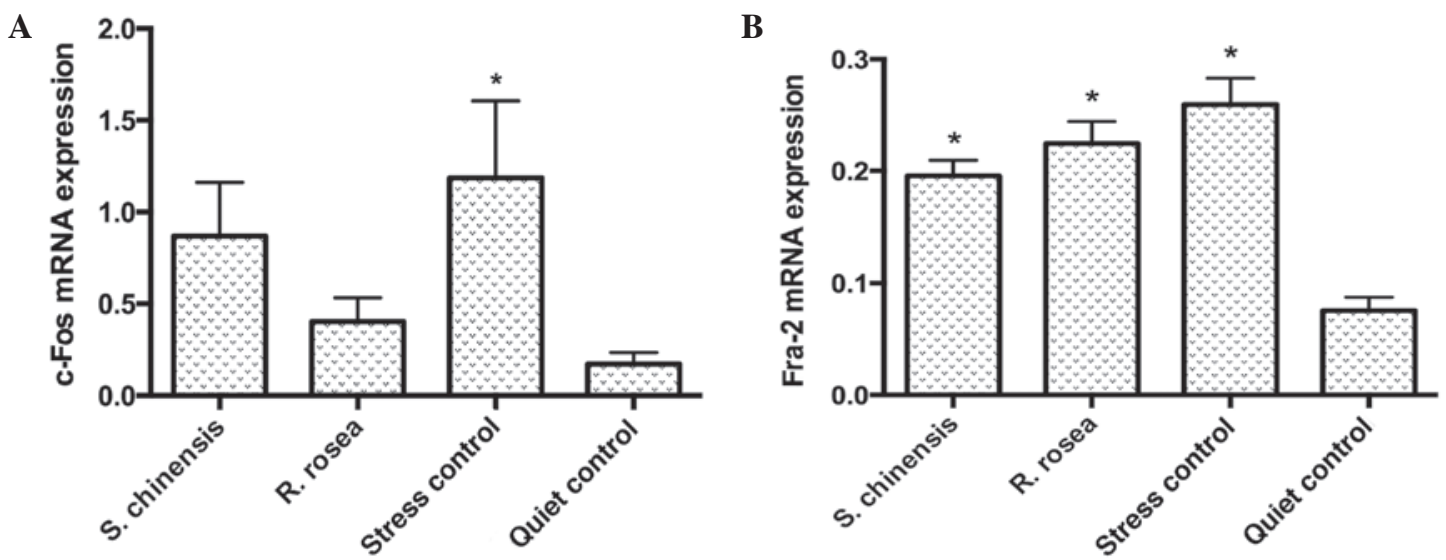

Figure 3. Comparison of c-Fos and Fra-2 mRNA expression levels in hypothalamus between the four groups. The mRNA expression levels of (A) c-Fos and (B) Fra-2 in the hypothalamus of the stress control group were significantly elevated compared with the quiet control group. The c-Fos mRNA expression levels in the $S$. chinensis and $R$. rosea groups showed no significant difference compared with quiet control group. Fra-2 expression in the $S$. chinensis and $R$. rosea groups showed no significant difference compared with the stress control group, while showing significant difference compared with the quiet control group. ${ }^{*} \mathrm{P}<0.05$ vs. the quiet control. S. chinensis, Schizandra chinensis; $R$. rosea, Rhodiola rosea.

addition, it has been reported that running training attenuates the ACTH response induced by physical or psychological stress $(33,34)$. In the present study, serum ACTH levels were evaluated after a total of $5 \mathrm{~h}$ exposure to psychological and physical stress. On the basis of the results of previous studies, it is not unexpected that the ACTH levels in the present study were not markedly increased, and decreased to a certain extent in the stress control group ( $<300 \mathrm{pg} / \mathrm{ml}$ on average) compared with the quiet control. In addition, a previous study has shown that blood concentrations of adrenal glucocorticoids increase to peak levels at 15-30 min and subsequently decline gradually to pre-stress levels at 60-90 $\mathrm{min}$ (35). This increase appears to be attenuated by repeated exposure to stress (36), which is consistent with the present result showing insignificant differences in CORT levels following the water-floating and treadmill exercise experiments. Therefore, this insignificance may be explained by the neural adaption caused by the previous repeated stress training during the first 6 days, indicating a possible mechanism of chronic stress characterized by reduced sensitivity of the HPA response to similar stressors, such as posttraumatic stress disorder (37-40).

Notably, the present results demonstrated that $S$. chinensis and $R$. rosea significantly decreased CRH mRNA expression levels $(\mathrm{P}<0.05)$ and the levels of serum CORT $(\mathrm{P}<0.05)$ in the rats exposed to water-floating and exhaustion experiments. $\mathrm{CRH}$ and CORT are pleiotropic, and exert effects via ubiquitously distributed intracellular receptors $(41,42)$. S. chinensis increased the mildly stress-reduced ACTH levels, and $R$. rosea showed a similar, but less marked effect. These results suggest that $S$. chinensis and $R$. rosea are able to reduce the changes in CORT and ACTH levels caused by prolonged and intense compound stress, thus balancing the HPA axis and maintaining homeostasis in rats.

In the present study, the effects of $S$. chinensis and $R$. rosea on the immune system were investigated by evaluating the serum levels of IL-1 $\beta$, which is a typical stress-associated cytokine and the most extensively studied inflammatory cytokine. The immune inflammatory response is considered to be closely associated with the stress system. Activation of the HPA axis produces marked inhibitory effects on the immune inflammatory response, as virtually all the components of the immune response are inhibited by cortisol (43). In turn, cytokines including TNF- $\alpha$, IL- $1 \beta$ and IL-6 stimulate the HPA axis independently, or in combination with one another (44). IL-1 is a critical mediator of adaptive stress responses, in addition to stress-associated neuropathology and psychopathology (45). A recent study demonstrated that the activation of the HPA axis by IL- $1 \beta$ is dependent on IL- 1 type 1 receptors in non-hematopoietic cells, such as brain endothelial cells, but not in perivascular macrophages (46). Furthermore, a 15-min swim stress has been shown to provoke a significant reduction in lipopolysaccharide (LPS)-induced IL-1 $\beta$ production at 120 and $240 \mathrm{~min}$ following LPS challenge $(<200 \mathrm{pg} / \mathrm{ml})$, compared with the control (>600 pg/ml) (47). In the present study, serum IL-1 $\beta$ levels in the rats were mildly, but not significantly, suppressed by compound stress. Treatment with $S$. chinensis and $R$. rosea appeared to increase the stress-reduced IL-1 $\beta$ levels. However, their effect on peripheral IL-1 $\beta$ levels was also mild and requires further investigation.

In addition to stress-induced changes in the HPA axis and cytokine expression following stimulation with various stressors, a number of 'molecular chaperones' appear to be crucially involved in the regulation of the neuroendocrine system and immune response (16). Stress-induced CRH expression causes ACTH release from the pituitary gland, and ACTH stimulates the release of adrenal hormones and NPY to mobilize energy and help the body to cope with stress, and in turn NPY activates the release of CRH $(18,48)$. Adaptogens are known to activate the expression of NPY in brain cells (49). Therefore, NPY production by brain cells has been suggested as a characteristic marker of the adaptogenic activity of plant extracts (50). Numerous studies have reported that acute and repeated physical stress may significantly increase the protein and mRNA expression levels of NPY in the amygdala $(17,51,52)$. In the present study, NPY expression in the hypothalamus following exposure to stress was investigated. The results showed that NPY mRNA expression levels in the hypothalamus were relatively low, with no statistically significant differences among the four groups. These results suggest that NPY expression in the hypothalamus does not have such a strong association with stress as does 
NPY expression in the amygdala. Further experiments are required to investigate the association between adaptogens and the production of NPY (protein and mRNA) in the amygdala after stress.

Numerous studies have identified the central pathways mediating the stress response by mapping neuronal activation using c-Fos as a dependable indicator $(36,53)$. Weinberg et al demonstrated that the kinetics of the c-Fos response to acute stimuli is transient (32). In their study, c-Fos gene expression in the prefrontal cortex (PFC), lateral septum (LS) and PVN peaked at $15 \mathrm{~min}$ of restraint, moderately declined at $60 \mathrm{~min}$ of restraint and had reduced by $180 \mathrm{~min}$ of continuous restraint; PVN c-Fos expression at 180 min of restraint remained increased compared with the expression levels in control rats; restraint for $60 \mathrm{~min}$ produced a significant increase in Fra- 2 mRNA expression in the PFC, LS and PVN, and by 180 min of restraint, Fra-2 expression returned to near-basal levels in all brain regions examined.

A previous study has demonstrated that stress induces a marked elevation in the expression levels of the AP-1 complex in the rat hypothalamus, pituitary gland, adrenal gland and gastric mucosa, suggesting that AP-1 binding may be a sensitive tool for monitoring the severity of inputs of extracellular stress signals (54). The protein Fra-2, which is a Fos-family member, competes with Fos protein for participation in the AP-1 transcription factor complexes. Each protein contributes different transactivation consequences to an AP-1 complex, leading to changes in the downstream expression of genes containing AP-1 elements in their promoter region $(24,55)$. The present results demonstrated that c-Fos and Fra-2 gene expression levels were significantly increased by stress, as demonstrated by the c-Fos and Fra- 2 mRNA expression levels remaining markedly elevated after the 5-h stress. In a previous study of seizure activity, Beer et al (26) observed the rapid induction of Fra mRNA by kainic acid and persisting levels after $6 \mathrm{~h}$, with prolonged Fra-2 protein expression for 7 days in the hippocampus. It has been reported that Fra- 2 protein levels in the adrenal medulla are significantly elevated after $2 \mathrm{~h}$ of a single or repeated immobilization stress, and are particularly pronounced at the termination of the immobilization, demonstrating the crucial transcriptional role of Fra-2 gene expression in the regulation of catecholamine biosynthetic enzymes in the stress response of the adrenal medulla (56).

In the present study, following persisting stimuli, Fra-2 mRNA expression was significantly induced in the hypothalamus after $5 \mathrm{~h}$ of physical and psychological stress. Furthermore, following treatment with $S$. chinensis or $R$. rosea, the compound stress-induced increase in c-Fos mRNA expression was attenuated, and was comparable with the levels in the quiet control rats. By contrast, Fra-2 mRNA expression remained unchanged at high levels following the administration of S. chinensis or $R$. rosea, and the effect of these herbs on the protein product of the Fra- 2 gene and Fos protein expression in the hypothalamus requires further investigation.

The stress-associated markers investigated in the present study are associated with each other, participating in the complex process of the stress response. For example, a synthetic analogue of an ACTH (4-10) fragment, known as Semax, has been shown to reduce stress-induced Fos protein expression in the PVN and medial septum of emotionally stressed rats (57). This may explain the observation in the present study that
ACTH levels were reduced and c-Fos levels increased in the stress control group compared with the quiet control group. As previously mentioned, IL-1 $\beta$ is able to independently stimulate HPA activity, leading to stress, potentially by enhancing the expression of c-Fos and CRH within the parvocellular division of the PVN (58).

In conclusion, the present study demonstrated that $\mathrm{CRH}$, c-Fos and Fra- 2 levels were significantly elevated after $5 \mathrm{~h}$ of prolonged compound stress. S. chinensis and $R$. rosea appear to exert anti-stress effects on stressed rats by balancing the HPA axis, and may reduce the expression of c-Fos in the hypothalamus. The present study had some limitations. As laboratory conditions were limited, alterations in the hypothalamic expression levels of proteins could not be investigated. The present study was also limited in that the whole hypothalamus was isolated, rather than one specific area; therefore, further studies are required to fully characterize the stress-protective effects of S. chinensis and $R$. rosea and to elucidate the mechanisms involved. These may include the analysis of Fos protein expression and its association with $\mathrm{CRH}$ production in hypothalamic neurons following treatment with $S$. chinensis or $R$. rosea and the potentially associated intracellular signaling pathways. The relatively moderate side effects and marked anti-stress effects of adaptogens indicate that they may provide an improved therapeutic option for the treatment of acute compound stress in the future.

\section{Acknowledgements}

The present study was supported by the Scientific Research Foundation in the Twelfth Five-Year Plan Period of China (no. CWS11J252). The authors thank the Department of Traditional Chinese Medicine of Nanjing Jinling Hospital for providing $S$. chinensis and $R$. rosea and Dr Guohong Wang in the Department of Radioimmunity of Nanjing Jinling Hospital for providing radioimmune analysis laboratory access.

\section{References}

1. Charmandari E, Tsigos C and Chrousos G: Endocrinology of the stress response. Annu Rev Physiol 67: 259-284, 2005.

2. Mills FJ: The endocrinology of stress. Aviat Space Environ Med 56: 642-650, 1985.

3. Tsigos C and Chrousos GP: Hypothalamic-pituitary-adrenal axis, neuroendocrine factors and stress. J Psychosom Res 53: 865-871, 2002.

4. Tsigos C and Chrousos GP: Physiology of the hypothalamic pituitary-adrenal axis in health and dysregulation in psychiatric and autoimmune disorders. Endocrinol Metab Clin North Am 23: 451-466, 1994.

5. Zorrilla EP, Logrip ML and Koob GF: Corticotropin releasing factor: A key role in the neurobiology of addiction. Front Neuroendocrinol 35: 234-244, 2014.

6. Koob GF: Corticotropin-releasing factor, norepinephrine, and stress. Biol Psychiatry 46: 1167-1180, 1999.

7. Wagner H, Nörr $\mathrm{H}$ and Winterhoff $\mathrm{H}$ : Plant adaptogens. Phytomedicine 1: 63-76, 1994.

8. Wiegant FA, Surinova S, Ytsma E, Langelaar-Makkinje M, Wikman G and Post JA: Plant adaptogens increase lifespan and stress resistance in C. elegans. Biogerontology 10: 27-42, 2009.

9. Mattioli L and Perfumi M: Rhodiola rosea L. extract reduces stress- and CRF-induced anorexia in rats. J Psychopharmacol 21: 742-750, 2007.

10. Panossian AG: Adaptogens: Tonic herbs for fatigue and stress. Altern Complement Ther 9: 327-331, 2003.

11. Panossian A and Wikman G: Pharmacology of Schisandra chinensis Bail.: An overview of Russian research and uses in medicine. J Ethnopharmacol 118: 183-212, 2008. 
12. Kelly GS: Rhodiola rosea: A possible plant adaptogen. Altern Med Rev 6: 293-302, 2001.

13. Kang YH and Shin HM: Inhibitory effects of Schizandra chinensis extract on atopic dermatitis in NC/Nga mice. Immunopharmaco Immunotoxicol 34: 292-298, 2012.

14. Brekhman II (ed): Chapter 3, Pharmacosanation Medicaments In: Man and Biologically Active Substances: The Effect of Drugs, Diet and Pollution on Health. 1st edition. Pergamon Press, New York, NY, pp49-59, 1980

15. Panossian A and Wagner H: Stimulating effect of adaptogens: An overview with particular reference to their efficacy following single dose administration. Phytother Res 19: 819-838, 2005

16. Panossian A, Wikman G, Kaur P and Asea A: Adaptogens exert a stress-protective effect by modulation of expression of molecular chaperones. Phytomedicine 16: 617-622, 2009.

17. Morales-Medina JC, Dumont Y and Quirion R: A possible role of neuropeptide Y in depression and stress. Brain Res 1314: 194-205, 2010

18. Morgan CA III, Rasmusson AM, Wang S, Hoyt G, Hauger RL and Hazlett G: Neuropeptide-Y, cortisol, and subjective distress in humans exposed to acute stress: Replication and extension of previous report. Biol Psychiatry 52: 136-142, 2002.

19. Karanikas E and Giouzepas I: Neuro-endocrinology of stress and immune mediated inflammation. Psychiatriki 19: 43-51, 2008 (In Greek)

20. Luckman SM,Dyball RE and Leng G: Induction of c-fos expression in hypothalamic magnocellular neurons requires synaptic activation and not simply increased spike activity. J Neurosci 14 4825-4830, 1994

21. Windle RJ, Kershaw YM, Shanks N, Wood SA, Lightman SL and Ingram CD: Oxytocin attenuates stress-induced c-fos mRNA expression in specific forebrain regions associated with modulation of hypothalamo-pituitary-adrenal activity. J Neurosci 24 : 2974-2982, 2004

22. Ryan KK, Mul JD, Clemmensen C, Egan AE, Begg DP, Halcomb K Seeley RJ, Herman JP and Ulrich-Lai YM: Loss of melanocortin-4 receptor function attenuates HPA responses to psychological stress. Psychoneuroendocrinology 42: 98-105, 2014.

23. Gagliano H, Delgado-Morales R, Sanz-Garcia A and Armario A: High doses of the histone deacetylase inhibitor sodium butyrate trigger a stress-like response. Neuropharmacology 79: 75-82, 2014

24. Kovács KJ: c-Fos as a transcription factor: A stressful (re)view from a functional map. Neurochem Int 33: 287-297, 1998.

25. Parrott RF and Vellucci SV: Stress-induced changes in c-fos immunoreactivity in the porcine brain. Br Vet J 150: 355-363, 1994

26. Beer J, Mielke K, Zipp M, Zimmermann M and Herdegen T: Expression of c-jun, junB, c-fos, fra-1 and fra-2 mRNA in the rat brain following seizure activity and axotomy. Brain Res 794: 255-266, 1998

27. Wang J, Zhao D, Li J, Wang G, Hu L, Shao J, Gu P, Du H and Wang Y: The impact of water-floating and high-intensity exercise on rat's HPA axis and interleukins concentrations. Acta Physiol Hung 99: 261-270, 2012.

28. Adamec R, Walling S and Burton P: Long-lasting, selective, anxiogenic effects of feline predator stress in mice. Physiol Behav 83: 401-410, 2004.

29. Cook DM, Kendall JW, Greer MA and Kramer RM: The effect of acute or chronic ether stress on plasma ACTH concentration in the rat. Endocrinology 93: 1019-1024, 1973.

30. Hauger RL, Millan MA, Lorang M, Harwood JP and Aguilera G: Corticotropin-releasing factor receptors and pituitary adrenal responses during immobilization stress. Endocrinology 123: 396-405, 1988.

31. Cam GR and Bassett JR: The plasma levels of ACTH following exposure to stress or nicotine. Arch Int Pharmacodyn Ther 264: 154-167, 1983.

32. Weinberg MS, Girotti M and Spencer RL: Restraint-induced fra-2 and c-fos expression in the rat forebrain: Relationship to stress duration. Neuroscience 150: 478-486, 2007.

33. Watanabe T, Morimoto A, Sakata Y, Tan N, Morimoto K and Murakami N: Running training attenuates the ACTH responses in rats to swimming and cage-switch stress. J Appl Physiol (1985) 73 2452-2456, 1992 .

34. Keller-Wood ME and Dallman MF: Corticosteroid inhibition of ACTH secretion. Endocr Revs 5: 1-24, 1984.

35. de Kloet ER, Joëls M and Holsboer F: Stress and the brain: From adaptation to disease. Nat Rev Neurosci 6: 463-475, 2005.

36. Chen $X$ and Herbert J: Regional changes in c-fos expression in the basal forebrain and brainstem during adaptation to repeated stress: Correlations with cardiovascular, hypothermic and endocrine responses. Neuroscience 64: 675-685, 1995.
37. Dhabhar FS, McEwen BS and Spencer RL: Adaptation to prolonged or repeated stress - comparison between rat strains showing intrinsic differences in reactivity to acute stress. Neuroendocrinology 65: 360-368, 1997.

38. Miller GE, Chen E and Zhou ES: If it goes up, must it come down? Chronic stress and the hypothalamic-pituitary-adrenocortical axis in humans. Psychol Bull 133: 25-45, 2007.

39. Seo JH, Kim TW, Kim CJ, Sung YH and Lee SJ: Treadmill exercise during pregnancy ameliorates post-traumatic stress disorder-induced anxiety-like responses in maternal rats. Mol Med Rep 7: 389-395, 2013.

40. O'Connor KA, Ginsberg AB, Maksimova E, Wieseler Frank JL, Johnson JD, Spencer RL, Campeau S, Watkins LR and Maier SF: Stress-induced sensitization of the hypothalamic-pituitary adrenal axis is associated with alterations of hypothalamic and pituitary gene expression. Neuroendocrinology 80: 252-263, 2004.

41. Axelrod J and Reisine TD: Stress hormones: Their interaction and regulation. Science 224: 452-459, 1984.

42. Munck A, Guyre PM and Holbrook NJ: Physiological functions of glucocorticoids in stress and their relation to pharmacological actions. Endocr Rev 5: 25-44, 1984.

43. Chrousos GP: Organization and integration of the Endocrine System. Sleep Med Clin 2: 125-145, 2007.

44. Chrousos GP: The hypothalamic-pituitary-adrenal axis and immune-mediated inflammation. N Engl J Med 332: 1351-1362, 1995.

45. Goshen I and Yirmiya R: Interleukin-1 (IL-1): A central regulator of stress responses. Front Neuroendocrinol 30: 30-45, 2009.

46. Matsuwaki T, Eskilsson A, Kugelberg U, Jönsson JI and Blomqvist A: Interleukin-1 $\beta$ induced activation of the hypothalamus-pituitary-adrenal axis is dependent on interleukin-1 receptors on non-hematopoietic cells. Brain Behav Immun 40: 166-173, 2014.

47. Connor TJ, Brewer C, Kelly JP and Harkin A: Acute stress suppresses pro-inflammatory cytokines TNF-alpha and IL-1 beta independent of a catecholamine-driven increase in IL-10 production. J Neuroimmunol 159: 119-128, 2005.

48. Haas DA and George SR: Neuropeptide Y-induced effects on hypothalamic corticotropin-releasing factor content and release are dependent on noradrenergic/adrenergic neurotransmission. Brain Res 498: 333-338, 1989.

49. Panossian A, Wikman G, Kaur P and Asea A: Adaptogens stimulate neuropeptide $\mathrm{Y}$ and $\mathrm{Hsp} 72$ expression and release in neuroglia cells. Front Neurosci 6: 6, 2012

50. Asea A, Kaur P, Panossian A and Wikman KG: Evaluation of molecular chaperons Hsp72 and neuropeptide Y as characteristic markers of adaptogenic activity of plant extracts. Phytomedicine 20: 1323-1329, 2013.

51. Sajdyk TJ, Fitz SD and Shekhar A: The role of neuropeptide Y in the amygdala on corticotropin-releasing factor receptor-mediated behavioral stress responses in the rat. Stress 9: 21-28, 2006

52. Sajdyk TJ, Johnson PL, Leitermann RJ, Fitz SD, Dietrich A, Morin M, Gehlert DR, Urban JH and Shekhar A: Neuropeptide $\mathrm{Y}$ in the amygdala induces long-term resilience to stress-induced reductions in social responses but not hypothalamic-adrenal-pituitary axis activity or hyperthermia. J Neurosci 28: 893-903, 2008.

53. Cullinan WE, Herman JP, Battaglia DF, Akil H and Watson SJ: Pattern and time course of immediate early gene expression in rat brain following acute stress. Neuroscience 64: 477-505, 1995.

54. Hinoi E, Fujimori S, Yoneyama M and Yoneda Y: Blockade by N-methyl-D-aspartate of elevation of activator protein-1 binding after stress in rat adrenal gland. J Neurosci Res 70: $161-171,2002$.

55. Karin M: The regulation of AP-1 activity by mitogen-activated protein kinases. J Biol Chem 270: 16483-16486, 1995.

56. Sabban EL, Liu X, Serova L, Gueorguiev V and Kvetnansky R: Stress triggered changes in gene expression in adrenal medulla: Transcriptional responses to acute and chronic stress. Cell Mol Neurobiol 26: 845-856, 2006

57. Umriukhin PE, Koplik EV, Grivennikov IA, Miasoedov NF and Sudakov KV: Gene c-Fos expression in brain of rats resistant and predisposed to emotional stress after intraperitoneal injection of the ACTH(4-10)analog - semax. Zh Vyssh Nerv Deiat Im I P Pavlova 51: 220-227, 2001 (In Russian).

58. Rivest $\mathrm{S}$ and Rivier C: Stress and interleukin-1 beta-induced activation of c-fos, NGFI-B and CRF gene expression in the hypothalamic PVN: Comparison between Sprague-Dawley, Fisher-344 and Lewis rats. J Neuroendocrinol 6: 101-117, 1994 